\title{
A NOTE ON LOCALLY COMPACT GROUPS
}

\section{A. M. GLEASON}

In this note we shall prove that every locally compact group can be embedded as a closed subgroup in a unimodular group. If the original group is locally Euclidean, the enlarged group will be also, hence the fifth problem of Hilbert is reduced to the unimodular case.

We shall use certain results concerning Haar measure whose proof may be found in A. Weil, L'integration dans les groupes topologiques et ses applications, Paris, Hermann, 1938, Chap. II, which we shall refer to as AW.

Let $\int$ stand for a left Haar integral. There is (AW) a continuous function $\Delta$ defined on $G$ such that $\int_{G} f\left(x s^{-1}\right) d x=\Delta(s) \int_{G} f(x) d x$ for all integrable functions $f$ and all $s \in G$.

Lemma. Let $G$ be a locally compact topological group and let $K$ be a closed normal unimodular subgroup such that $G / K$ is also unimodular. If $s \in G$ the automorphism of $K$ defined by $k \rightarrow s^{-1} k s$ multiplies the Haar measure of $K$ by $\Delta(s)$.

Proof. Let $s$ be fixed and let $\mu$ be a left Haar measure on $K$. Because of unicity properties of Haar measure, there is a constant $c$ such that, for all measurable sets $E, \mu\left(s^{-1} E s\right)=c \mu(E)$; we must show that $c=\Delta(s)$. The first formula implies that, for any integrable function $f$, $\int_{K} f(k) d\left(s^{-1} k s\right)=c \int_{K} f(k) d k$, which may be abbreviated by the formula $d\left(s^{-1} k s\right)=c d k$.

Let $f$ be a non-negative continuous function on $G$ which vanishes outside a compact set but is not everywhere zero. If the integrals are suitably normalized, we have (AW) $\int_{G} f(x) d x=\int_{G / K} F(X) d X$, where $x \rightarrow X$ is the natural map of $G$ onto $G / K$ and $F(X)=\int_{K} f(x y) d y$, the latter being independent of the choice of representative $x$ of $X$. Let $f^{*}(x)=f\left(x s^{-1}\right)$. Putting $\quad F^{*}(X)=\int_{K} f^{*}(x y) d y=\int_{K} f\left(x y s^{-1}\right) d y$ $=\int_{K} f\left(x s^{-1} k\right) d\left(s^{-1} k s\right)=c \int_{K} f\left(x s^{-1} k\right) d k=c F\left(X S^{-1}\right)$, we have

$$
\begin{aligned}
\int_{G} f\left(x s^{-1}\right) d x & =\int_{G} f^{*}(x) d x=\int_{G / K} F^{*}(X) d X=c \int_{G / K} F\left(X S^{-1}\right) d X \\
& =c \int_{G / K} F(X) d X=c \int_{G} f(x) d x
\end{aligned}
$$

(recalling that $G / K$ is unimodular), which shows that $c=\Delta(s)$ since $\int_{G} f(x) d x$ is not zero.

Received by the editors June 11, 1948. 
THEOREM. A locally compact group $G$ is isomorphic to a closed subgroup of a unimodular locally compact group whose space is $G \times R$, where $R$ is the real line.

Proof. The function $\Delta$ is a homomorphism of $G$ into the positive real numbers under multiplication since $\Delta(s t)=\Delta(s) \Delta(t)$. The kernel $K$ of this homomorphism is a unimodular normal subgroup of $G$ (AW) and $G / K$ is abelian and hence unimodular. Applying the lemma we obtain $d\left(g k g^{-1}\right)=\Delta\left(g^{-1}\right) d k$ for $k \in K$ and $g \in G$.

Let $H=G \times R$ as a topological space; then $H$ is locally compact. Define a binary operation in $H$ by setting $\left(g_{1}, r_{1}\right)\left(g_{2}, r_{2}\right)=\left(g_{1} g_{2}\right.$, $\left.r_{1} \Delta\left(g_{2}^{-1}\right)+r_{2}\right)$. This is easily verified to be associative; there is a unit element $(e, 0)$; the element $(g, r)$ has the inverse $\left(g^{-1},-r \Delta(g)\right)$; and both the operation and its inverse are continuous: hence $H$ is a locally compact topological group. Since $G \times\{0\}$ is a closed subgroup of $H$ isomorphic to $G$, the proof will be complete if we show that $H$ is unimodular.

The set $L$ of all pairs $(k, r)$, where $k \in K$, is a closed subgroup of $H$. Since $\Delta(k)=1$ for all $k \in K, L$ is the direct product of the groups $K$ and $R$. The Haar measure of $L$ is the product of the Haar measures of $K$ and $R$, which we may symbolize by the formula $d(k, r)=d k d r$. Since both these groups are unimodular, $L$ is unimodular. Let $(g, x)$ be a fixed element of $H$, and let $(k, r) \in L$. Then $(g, x)(k, r)(g, x)^{-1}$ $=\left(g \mathrm{~kg}^{-1}, r \Delta(g)\right) \in L$. Hence $L$ is normal. Moreover, the automorphism of $L$ induced by $(g, x)$ is measure preserving since $d\left(\mathrm{gkg}^{-1}, r \Delta(g)\right)$ $=d\left(g k g^{-1}\right) d(r \Delta(g))=\Delta\left(g^{-1}\right) d k \Delta(g) d r=d k d r=d(k, r)$. The group $H / L$ $(\approx G / K)$ is abelian and hence unimodular. Applying the lemma, we see that $H$ is unimodular.

HARVARD UNIVERSITY 